EPJ Web of Conferences 47, 02003 (2013)

DOI: $10.1051 /$ epjconf/20134702003

(C) Owned by the authors, published by EDP Sciences, 2013

\title{
Small flow rate can supply inwardly migrating shortest-period planets
}

\author{
S.F. Taylor ${ }^{1,2, a}$ \\ ${ }^{1}$ Participation Worldscope/Global Telescope Science, Hong Kong, SAR China and Sedona, \\ Arizona, USA \\ 2Job Seeking
}

\begin{abstract}
The number of exoplanets found with periods as short as one day and less was surprising given how fast these planets had been expected to migrate into the star due to the tides raised on the star by planets at such close distances. It has been seen as improbable that we would find planets in such a small final fraction of their lives [1]. The favored solution has been that the tidal dissipation is much weaker than expected, which would mean that the final infall would be a larger fraction of the planets' life. We find no reason, however, to exclude the explanation that a small number of planets are continuously sent migrating inwards such that these planets indeed are in the last fraction of their lives. Following the observation that the distribution of medium planets disfavors tidal dissipation being significantly weaker than has been found from observations of binary stars [2], we now show that the numbers of planets in such a "flow" of excess planets migrating inwards is low enough that even depletion of the three-day pileup is a plausible source. Then the shortest period occurrence distribution would be shaped by planets continuously being sent into the star, which may explain the depletion of the pileup in the Kepler field relative to the solar neighborhood [3]. Because Kepler observes above the galactic plan, [3] suggested the Kepler field may include an older population of stars. The tidal dissipation strength in stars due to giant planets may be not greatly weaker than it is in binary stars.
\end{abstract}

\section{INTRODUCTION}

The discovery of too many planets found in the last fraction of their lifetimes is among the most unexplained exoplanet discoveries [1]. Either tidal migration is slower than expected, or these planets have recently been sent inward. The favored explanation has been that tidal dissipation within the star from tides on the star raised by these planets is much weaker than the tidal dissipation as measured from binary star statistics [1], but we show the more plausible explanation is that more planets migrate inwards than expected. It is thought that inwardly migrating planets circularize and nearly cease inward migration, avoiding infall into the star. We propose that planets in the pileup may be caused to migrate more rapidly than expected than if most orbits are circular.

We show that the discrepancy between the inferred tidal strength found by $[2,4]$ is also supported by directly calculating the infall times for the Kepler planet candidates (hereafter "planets").

\section{INWARD MIGRATION AS SOURCE OF SHORTEST PERIOD PLANETS}

The strength of dissipation had been expected to be stronger than a tidal dissipation strength " $Q_{*}^{\prime}$ " of $10^{7}$, which is expressed as $Q_{*}^{\prime}$ being "less than" $10^{7}$, where the dissipation strength is proportional to

\footnotetext{
ae-mail: astrostuart@gmail.com
}

This is an Open Access article distributed under the terms of the Creative Commons Attribution License 2.0, which permits unrestricted use, distribution, and reproduction in any medium, provided the original work is properly cited. 


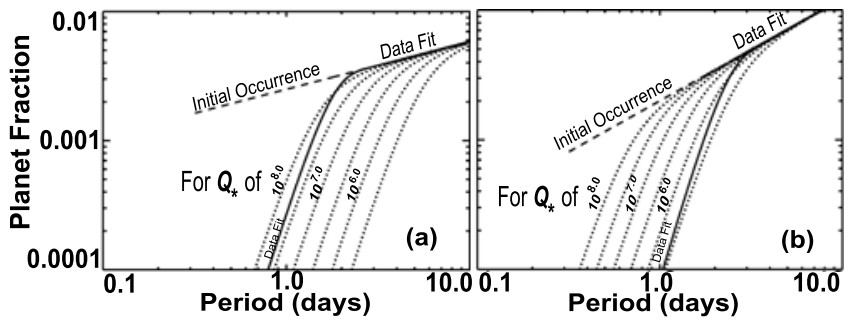

Figure 1. Migrated evolution compared to data for masses and radii for an example age of $4.5 \mathrm{Gyr}$ for planets with masses summed over the ranges given in the text, (a) for "large" planets, and (b) for "medium" planets.

$1 / Q_{*}^{\prime}$. However, careful studies based on the evolution of the occurrence distribution have presented planet distribution to be consistent with $Q_{*}^{\prime}$ weaker than $10^{7}$ (e.g., [5]), when assuming no input of new planets migrating inwards. We find that the rate of planet migrating inwards need only be on the order of $10^{-12}$ or less planets per year in order for the presence of the planets with these shortest periods to be consistent with a tidal dissipation $Q_{*}^{\prime}$ strenght of $10^{7}$ or stronger. This number is low enough to be supplied by a decay in the long observed three day pileup of giant planets, or it could be due to a hypothesized flow of planets from further out. Both explanations have support: The number of planets in the pileup of planet candidates (hereafter "planets") found by Kepler has been reported to be $40 \%$ lower than for the planets found in the solar neighborhood by [3], who suggest that perhaps the Kepler field, being above the galactic plane, has an older population of stars. The pileup may have decayed. The hypothesis of a flow of "high eccentricity migration" (HEM) of giant planets supplying the pileup has been suggested by [6], though such eccentric planets have not yet been found [7].

The shortest period distribution of planets has a fall off that within large uncertainty is consistent with migration due to tides in the star caused by the planet [2]. Tidal migration due to these "stellar" tides produce an occurrence distribution with a fall off that has a power index of $13 / 3$ based on equations of [8]. The two-power law fit to the Kepler planet occurrence distribution by [3] gives a fall off in the shortest period region that for giant and medium radii planets is reasonably close to $13 / 3$. We obtain the power index of the fall off from the fit by [3], by suming their two indices $\beta+\gamma$, which is the power index of their fit function in the limit of the period going to zero. We call the three ranges in radii used by [3] large, medium and (relatively) small. We assign the following radii and mass ranges to use in calculations of tidal migration, in earth radii $R_{\oplus}$, and earth masses, $M_{\oplus}$ : "Large" planets of radii from 8 to $16 R_{\oplus}$ and of mass from 100 to $2000 M_{\oplus}$, "medium" planets of radii 4 to $8 R_{\oplus}$ and of mass from 100 to $2000 M_{\oplus}$, and "small" planets of radii 2 to $4 R_{\oplus}$ and of mass from 10 to $100 M_{\oplus}$. The power indices found by [3], for giant planets, $4.5 \pm 2.5$, and for medium planets, $4.8 \pm 1.3$, are both consistent with the value of $13 / 3$ indicating currently inwardly tidally migrating planets, but the power index for superearth planets, $2.9 \pm 0.4$, is too low to be from ongoing tidal migration. The slope of the superearth planets likely originated with their formation.

We present the evolution of the fall off as a function of $Q_{*}^{\prime}$ for a summed range of stellar ages in Fig. 1. We calculate tidal migration operating on an initial occurrence distribution of a single power law to produce a distribution with a fall off, giving the two-power law distribution that resembles the actual distribution. The result, as shown in Fig. 1 is similar to the two-power law distribution of [3].

In Fig. 1, we compare fits by [3] with fall offs calculated for a range of tidal distributions. We show the occurrence distributions of [3] for giant and medium radii planets in Fig. 1, plotted against our calculations of occurrence distributions summed for mass after a representative migration time of 4.5 Gyr, shown for several values of tidal dissipation strengths $Q_{*}^{\prime}$. The fall off for giant and medium planets could be interpreted as giving different values of $Q_{*}^{\prime}$ for differently sized planets, but it could 


\section{Hot Planets and Cool Stars}
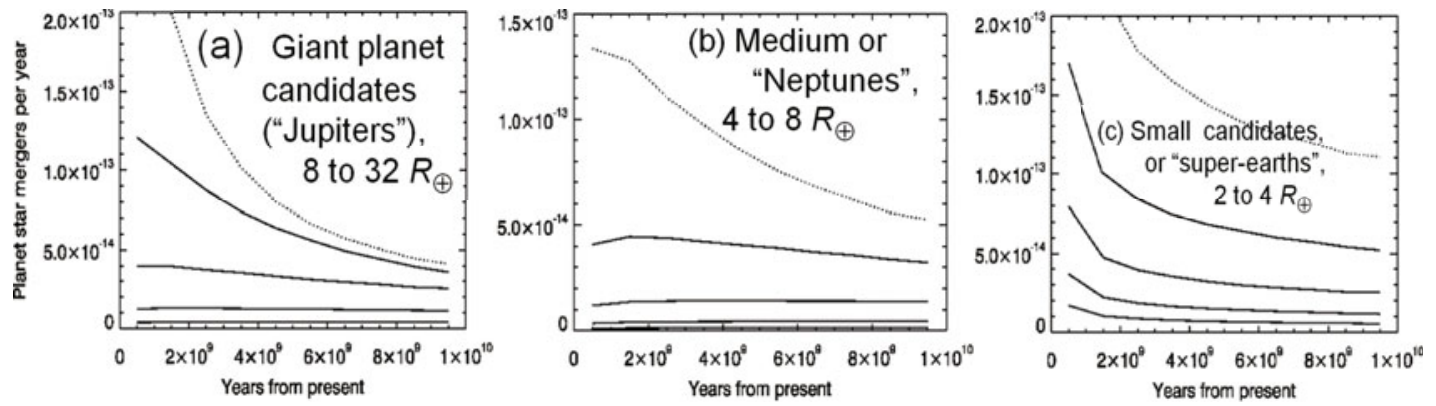

Figure 2. Future fall in rates for the three ranges of planet radii (panels for large, medium, and small radii) each with plots for tidal dissipation values of $\log \left(Q_{*}^{\prime}\right)$ values of $10^{6.5}$ (dotted, top), $10^{7.0}, 10^{7.5}, 10^{8.0}$, and $10^{8.5}$. Rate of fall in calculated based on fit.

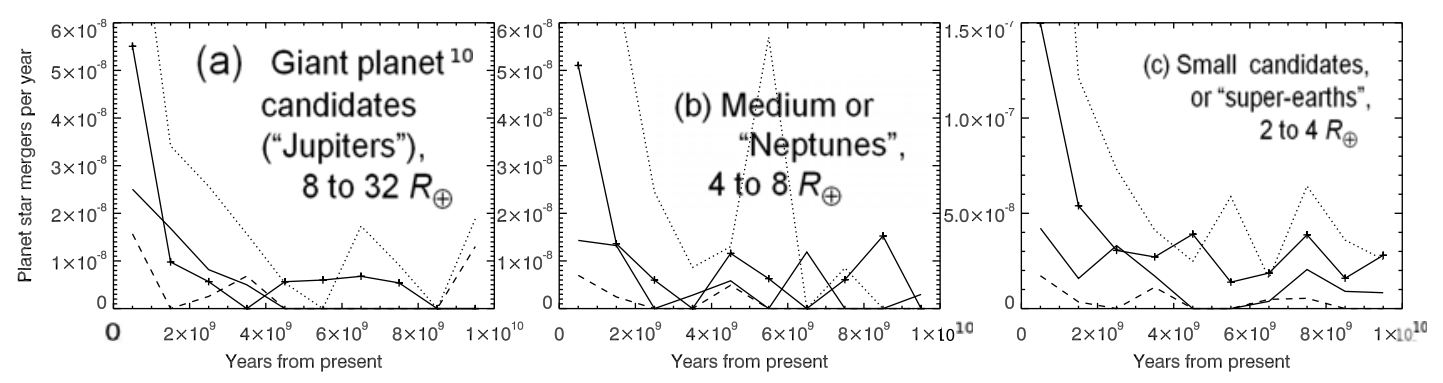

Figure 3. Rate of fall in calculated by modeling when Kepler candidates would fall in, with the same range of values as in Fig. 2.

also be explained by more giant planets migrating inwards. The difference appears to correspond with the radii where the pile up of giant planets occurs, which suggests that the pile up is related to the higher occurrence of the shortest period giant planets. This difference appears to be best reconciled by a larger rate (flow) of planets migrating from further out. This may or may not be related to a more distant HEM flow of giant planets that [6] propose may have supplied at least a part of the pileup, because depletion of the pileup may be sufficient.

In the three panels of Fig. 2 we show for the three size ranges of planets the calculated future infall rates plotted for stellar tidal dissipation values of $Q_{*}^{\prime}$ from $10^{6.5}$ to $10^{8.5}$, using the fits of [3] as the initial occurrence distributions. We also show infall calculated directly using data directly in Fig. 3, where we see the same discrepancy, though the result is noisy. The correct value of $Q_{*}^{\prime}$ is the one that gives infall rates that decrease at a rate no faster than the supply of planets decreases as stars age. The only way to maintain the presence of more giant than medium planets in the shortest period range is if there is a larger inward flow of giant planets than for medium planets. (This would only start adding to the infall after the new planets migrate in). Arriving planets would produce a flatter curve for $Q_{*}^{\prime}$ closer to $10^{7}$.

Inward planet migration might cause pollution of the star if other planets orbits are disrupted. The correlation between stellar $\mathrm{Fe} / \mathrm{H}$ and eccentricity presented in Fig. 3 of [4] suggests that high eccentricity planets could be associated with recent pollution. For systems found by radial velocity with periods less than 200 days (exoplanets.org), we find a less than $3 \%$ chance that $\mathrm{Fe} / \mathrm{H}$ values for stars hosting planets with orbits of eccentricity above and below 0.35 represent the same population. 


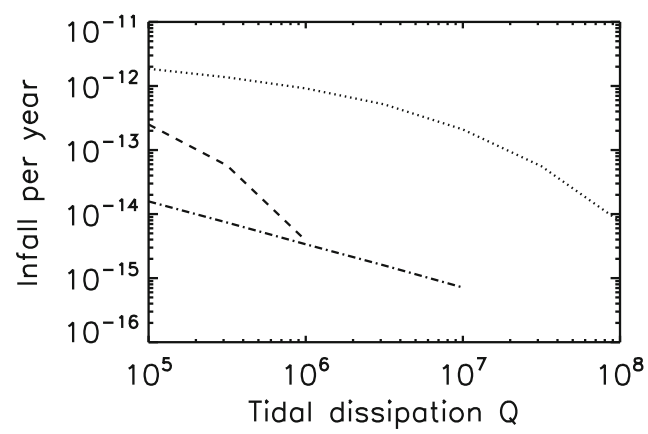

Figure 4. The difference in planet infall rate per year as a function of $Q_{*}^{\prime}$ between the flow at 1 and $10 \mathrm{Gyr}$, shown for three radii ranges. This shows that the likely rate of inward flow of giant planets (dotted) is higher than that for medium (dashed) and smaller (dot-dashed) planets. No line is shown for $Q_{*}^{\prime}$ values not needing a flow. It takes only this rate of inward planet flow to maintain a constant future infall rate.

\section{SMALL SUPPLY REQUIRED - PILEUP DEPLETION PROBABLY SUFFICIENT}

We find that the rate of planet infall required to provide the shortest period planet population is small enough to reasonably expect that depletion of the pileup or a migration of planets from further out could supply enough planets to explain short period planets continually infalling. We show the rates required in Fig. 4, where we have taken the difference in the rates of infall (as in Fig. 2) at 1 and 10 Gyr. The rate of this flow only needs to be less than $10^{-12}$ giant planets per year per star for the fall in rates to be consistent.

The $40 \%$ smaller size in the pileup in the Kepler field, [3], compared to ground surveys that predominantly sample the solar neighborhood [9] could indicate that the pileup decreases with age. This decrease is likely large enough to supply the small ongoing infall of planets migrating into the star. It is important to study whether planets in the pileup region might be having their eccentricity pumped up, or might be disrupted from planets migrating from further out, decreasing the numbers of planets in the pileup.

The first sign of stronger tidal migration could come in only a few years, when enough time has elapsed to measure decreases in the shortest period planets [1]. An important measure of the rate of planet infall will be observing transients from planet/star mergers, which will likely have sufficient luminosity to be observable from nearby galaxies by upcoming or current transient surveys [10, 11].

\section{References}

[1] Hamilton, D.P., Nature 460, 1086, (2009)

[2] Taylor, S.F. arXiv:astro-ph/1206.1343 (2012a)

[3] Howard, A.W., Marcy, G.W., \& Bryson, S.T. et al., ApJS 201, 15 (2012)

[4] Taylor, S.F. arXiv:astro-ph/1211.1984 (2012b)

[5] Penev, K., Jackson, B., Spada, F. \& Thom, N., Ap.J. 751, 96 (2012)

[6] Socrates, A., Katz, B., Dong, S. \& Tremaine, S., ApJ 750, 106 (2012)

[7] Dawson, R.I., Murray-Clay, R.A., \& Johnson, J.A., arXiv:astro-ph/1211.0554 (2012)

[8] Jackson, B., Barnes, R. \& Greenberg, R., ApJ, 698, 1357 (2009)

[9] Marcy, G., Butler, R. P., Fischer, D., et al., PTPS 158, 24 (2005)

[10] Taylor, S.F. arXiv:1009.4221, (2010)

[11] Metzger, B.D., Giannios, D. \& Spiegel, D.S., MNRAS, 425, 27 (2012) 